\title{
Поиск оптимальной стратегии для выхода на новые рынки леса и пиломатериалов
}

\author{
Родион Рогулин* , Ева Пугачёва, Владислав Матвеев, \\ Валерий Максименко, Владимир Жандармов, Данила Рогулин
}

Дальневосточный федеральный университет, г. Владивосток, Россия

Владивостокский государственный университет экономики и сервиса, г. Владивосток, Россия

\author{
Информация о статье \\ Поступила в редакичию: \\ 14.07.2019 \\ Принята \\ к опубликованию: \\ 25.10.2019 \\ УДК 338.28 \\ JEL C60
}

\section{Ключевые слова:}

математическое моделирование, линейное программирование, максимальный поток, размещение центров, производство

Keywords:

mathematical modeling, linear programming, maxi- mum flow, placement of centers, production

\begin{abstract}
Аннотация
Предложено комплексное решение трех задач линейного программирования: производственной (классическая постановка), задачи размещуения изентров, а такэе максимального потока. Подобные задачи в предложенной комплексной постановке часто возникают на предприятиях в прочессе производства и реализации продукции. Рассмотрены основнье алгоритмы поиска оптимального решения, сформулирована комплексная задача, построена модель и реализован алгоритм решения, проведено сравнение существующего метода и авторского. Предложенная модель может быть использована на любом предприятии, где необходимо найти оптимальный комбинаторный вариант для производства с цуелью минимизации производственных издержек и затрат на транспортировку готовой продукции, а также получения максимальной прибыли.
\end{abstract}

\section{Finding the best Exit Strategy to New Wood and Lumber Markets}

Rodion Rogulin, Eva Pugacheva, Vladislav Matveev, Valerii Maksimrnko, Vladimir Zhandarmov, Danila Rogulin

\begin{abstract}
This article proposes a comprehensive solution to three linear programming problems: Production problem (classical formulation), Center allocation problem, Maximum flow problem. Similar tasks in the proposed complex formulation often arise at enterprises in the process of production and sale of products. The basic algorithms for finding the optimal solution are considered, a comprehensive problem is formulated, a model is built, and a solution algorithm is implemented, the existing method and the author are compared. The proposed model can be used at any enterprise where it is necessary to find the optimal combinatorial option for production in order to minimize production costs and the cost of transporting finished products, as well as maximize profits. This task is exactly suitable for the economic situation,
\end{abstract}

* Автор для связи: rafassiaofusa@mail.ru DOI: https://dx.doi.org/10.24866/2311-2271/2019-3/40-47 
when the enterprise has yet to enter the market, and it makes attempts to determine the places of production from the list and tries to send as many goods as possible in order to occupy its niche in the market. The task that we are solving for the first time appeared at a timber processing enterprise. Such a problem is nontrivially combinatorial.

\section{Введение}

Каждое предприятие в ходе хозяйственной деятельности ставит главной задачей максимизацию прибыли при ограничениях на ресурсы. При всем многообразии методов оптимизации процессов управления ресурсами предприятий, в научной литературе недостаточно представлены единые алгоритмы и модели для нахождения оптимального решения комплексных проблем хозяйственной деятельности предприятия. В статье рассмотрены 3 основные задачи компаний: задача производства (оптимальный выпуск продукции), задача размещения центров, задача учета времени.

При имеющихся запасах ресурсов и заданных рынком ценах, необходимо найти оптимальный объем при дополнительном условии наличия норм затрат ресурсов на производство единицы продукции. Такая задача получила название производственной [1].

Вторая задача, не менее сложная с точки зрения трудозатрат на ее решения [2], подразумевает решение вопросов об определении мест производства из заранее определенных к рассмотрению районов. Ограничения описаны в [4].

Задача максимального потока заключается в максимизации начальных входных на граф потоки, а ограничением выступает равенство потоков и максимальная пропускная способность графа. Рассмотрим ряд методов и моделей, которые решают данные задачи (табл. 1).

Таблииа 1

Методы и модели решения поставленных задач

\begin{tabular}{|c|c|}
\hline Методы и модели \Факторы & Описание стратегии \\
\hline Линейное программирование (ЛП) [5] & $\begin{array}{l}\text { Каждая подзадача составляется и решается, как отдельная задача } \\
\text { ЛП, кроме задачи учета времени - она является дополнением к ограничениям. }\end{array}$ \\
\hline $\begin{array}{l}\text { Квадратическое программирование (КП) } \\
\text { [6] }\end{array}$ & $\begin{array}{l}\text { Составляется отдельная квадратическая модель, после работы стан- } \\
\text { дартных алгоритмов КП, представляется ответ к задаче в виде одномерного мас- } \\
\text { сива. }\end{array}$ \\
\hline Supply Chain Management (SCM) [7] & $\begin{array}{l}\text { Управленческая концепция и организационная стратегия, заключающаяся в } \\
\text { интегрированном подходе к планированию и управлению всем потоком инфор- } \\
\text { мации о сырье, материалах, продуктах, услугах, возникающих и преобразую- } \\
\text { щихся в логистических и производственных процессах предприятия, нацелен- } \\
\text { ном на измеримый совокупный экономический эффект (снижение издержек, } \\
\text { удовлетворение спроса на конечную продукцию). Концепция основана на гене- } \\
\text { тическом алгоритме. }\end{array}$ \\
\hline Генетический алгоритм [8] & $\begin{array}{l}\text { Эвристический алгоритм поиска, который используется для поиска решения } \\
\text { задач оптимизации и моделирования. Стратегия заключается в случайном под- } \\
\text { боре, комбинирования и вариаций изначальных параметров с использованием } \\
\text { механизмов, аналогичных естествен- ному отбору в природе. Является разно- } \\
\text { видностью эволюционных вычислений, с помощью которых решаются оптими- } \\
\text { зационные задачи с использованием методов естественной эволюции, таких как } \\
\text { наследование, мутации, отбор и кроссинговер. Отличительной особенностью } \\
\text { генетического алгоритма является акцент на использование оператора «скрещи- } \\
\text { вания», который производит операцию рекомбинации решений-кандидатов, } \\
\text { роль которой аналогична роли скрещивания в живой природе. }\end{array}$ \\
\hline
\end{tabular}

Источник: составлено авторами 
Для решения каждой из вышеперечисленных задач существуют отдельные модели $[9,10,11]$, но мы предлагаем комплексное решение, подразумевающее единую линейную модель смешано-целочисленного программирования для 3-х проблем лесоперерабатывающего комплекса.

Сформулируем обобщенную постановку задачи: каков объем производства продукции при заданных объемах ресурсов на складе, данных о максимальном потоке каждой дуги графа - дороги, данных о стоимости открытия пункта производства из заранее отобранных возможных районов расположения. Цель: максимизировать доход от продажи, объем перевозок по графу и минимизировать издержки в процессе открытия производства (*).

Для решения вышеперечисленных задач используются алгоритмы поиска оптимального решения - метод отсечения (Гомори) [15].

\section{Математическая модель}

Пусть существует сетка трудозатрат производства для производства каждого вида товара из исходного вида сырья. Обозначим саму сетку как

$$
A=\left\{A_{i j}\right\}, i=1: n_{1}, j=1: m_{1},
$$

где это элемент, соответствующий тому, сколько потребуется ресурса $\mathrm{i}$ для производства ј товара. Пусть также существует граф дорог (матрица смежности) с ее пропускной способностью и обозначим ее как

$$
D=\left\{d_{i_{1} j_{1}}\right\}, i_{1}=1: n, j_{1}=1: n \text {. }
$$

Определим вектор цен реализации товара $\mathrm{j}$, как

$$
P=\left\{p_{j}\right\}_{,} j=1: m_{1}
$$

Пусть известны затраты на открытие склада. Обозначим их как Определим вместимость складов как

$$
L=\left\{l_{j}\right\}_{j}=1: m_{1},
$$

Для полноты набора данных остается определить количество запасов сырья, обозначим их как

$$
b=\left\{b_{i}\right\}, i=1: n_{1}
$$

Зададим параметр $Q$, как параметр, отвечающий за максимальное количество открытых складов. Пусть - есть количество товара, перевозимое из пункта $i_{1}$ в пункт $k_{j}, \quad-$ произведенное количество $j$ товара, $-j$ пункт производства - разновидность ресурсов, - разновидность товара, - вершин в графе.

Математическая модель задач: максимального потока описана в $[1,2,3]$, производственной описана в $[1,2,3]$, размещения центров описана в $[1,2,3]$.

Объединим три вышеописанные математические модели.

Производство не может произвести больше, чем у него есть на то ресурсов, обозначим следующим ограничением:

$$
\sum_{j=1}^{m_{1}} A_{i j} k_{j} \leq b_{i}, i=1: n_{1}
$$

Количество открытых пунктов не должно превышать Q, напишем ограничение в виде:

$$
\sum_{j=1}^{m_{1}} z_{j} \leq Q
$$




$$
\text { где } z_{j} \in\{0 ; 1\} \text {. }
$$

Вместимость пунктов производства не должно превышать L, тогда запишем ограничение ниже:

$$
k_{j} \leq l_{j}, j=1: m_{1}
$$

Объем вывоза не должен превышать объем производства в каждом пункте, обозначим это как:

$$
\sum_{j_{1}=1}^{n} x_{j j_{1}} \leq k_{j} j=1: m_{1}
$$

Объем входящий в вершину должен быть равным объему, выходящему из этой же вершины, запишем это ниже, как:

$$
\sum_{i_{1}=1}^{n} x_{i_{1} j_{1}}=\sum_{j_{1}=1}^{n} x_{i_{1} j_{1}} ; i_{1}, j_{1}=1: n
$$

Объем, проходящий по дуге, не должен превышать ее пропускную способность, обозначим как:

$$
0 \leq x_{i_{1} j_{1}} \leq d_{i_{1} j_{1}} ; i_{1}, j_{1}=1: n
$$

Запишем (*) в виде целевой функции, как:

$$
\sum_{j_{1}=1}^{n} \sum_{i_{1}=1}^{n} x_{i_{1} j_{1}}+\sum_{j=1}^{m_{1}} k_{j} p_{j}-\sum_{j=1}^{m_{1}} z_{j} f_{j} \rightarrow \max
$$

Объединяя формулы 7-13 в единую систему получаем задачу $F_{0}$ линейного целочисленного программирования (смешанно-целочисленного).

Задача $F_{0}$ решена с помощью пакета Matlab. Ответ получим в виде одномерных массивов $\mathrm{X}$. Размерность $\mathrm{X}=2 r+n^{2}$. Первые $\mathrm{r}$ элементов отвечают за количество произведенного товара. Следующие $n^{2}$ переменных объем перевезенной продукции по каждой дуге. Последние элементов өтвечают за значения вспомогательных переменных. Рассмотриу ее подробнее.

$\mathrm{B}$ нашем примере количество пунктов производства $\mathrm{r}=16$. Пусть $\mathrm{M}-$ число вершин графа, тогда M-r есть число остальных вершин (перекрестки, склады, перевалочные пункты, пункты потребления и т.д.)

\section{Пример}

Пусть даны матрицы норм затрат,

$$
A=\left(\begin{array}{llllllllllllllll}
1 & 2 & 6 & 2 & 4 & 5 & 6 & 7 & 1 & 2 & 6 & 2 & 4 & 5 & 6 & 7 \\
2 & 6 & 9 & 4 & 5 & 3 & 2 & 6 & 2 & 6 & 9 & 4 & 5 & 3 & 2 & 6 \\
5 & 4 & 5 & 2 & 1 & 7 & 1 & \$ D & 5 & 4 & 5 & 2 & 1 & 7 & 1 & 5 \\
4 & 8 & 9 & 8 & 3 & 2 & 4 & 5 & 5 & 4 & 5 & 2 & 1 & 7 & 1 & 5
\end{array}\right)
$$

пропускной способности графа $D$, затраты на открытие $f$, матрицы цен $P$, запасов ресурсов, вместимость складов. Все данные представлены в [3]. На рис. 1 можно увидеть произвольную визуализацию. Номера вершин - пункты производства, промежуточные пункты, пункты потребления. Веса дуг матрицы есть максимальное число единиц продукции, которое можно провезти по каждой дуге. 


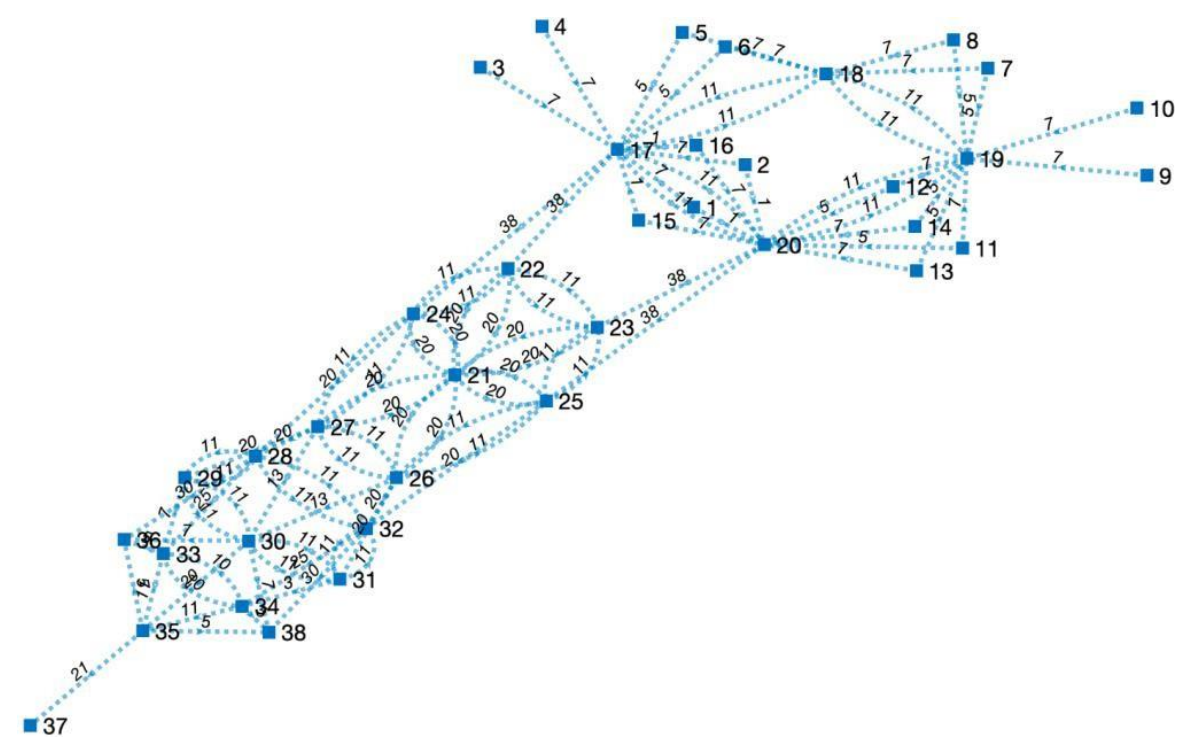

Рuc. 1. Произвольная визуализация матрицы D

Источник: составлено авторами

На рис. 2 показан оптимальный вариант для вывоза продукции.

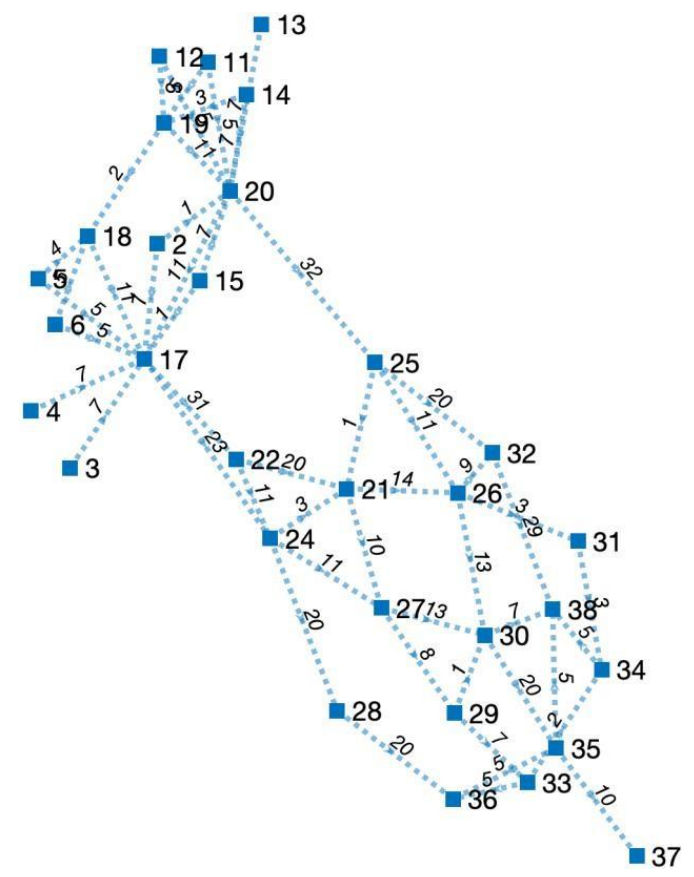

Puc. 2. Визуализация графа перевозок при решении комплексной задачи Источник: составлено авторами

В табл. 3 представлены выходные данные программных реализаций [3]. 
Таблица 3

Сравнение методов решения поставленной задачи описанными выше метода-

\begin{tabular}{|l|l|}
\hline \multicolumn{1}{|c|}{ Параметр $\backslash$ метод } & \multicolumn{1}{c|}{ Комплексно } \\
\hline Объем произведенной продукции (вектор), шт & $(0,8,7,9,10,0,0,0,0,10,10,7,10,8,0)$ \\
\hline Остатки сырья (вектор), шт & $(36,175,385,615)$ \\
\hline Прибыль, у. е. () & 7,0850 \& $10^{22}$ \\
\hline
\end{tabular}

Источник: составлено авторами

\section{Выводы}

В данной статье была рассмотрена одна из возможных постановок задачи, которая обобщает ранее известные 3 классические задачи линейного программирования. Было показано, что такую задачу возможно сформулировать в рамках задачи линейного программирования. Решен пример на 38 вершинах с 16 пунктами входа, 3 пунктами выхода. Показано, что такую задачу возможно решать и визуализировать средствами пакета Matlab. Pacсмотрен ряд возможных добавлений ограничений в модель. Такая постановка задачи и модель могут быть использованы на любом предприятии, где необходимо найти оптимальный комбинаторный вариант для получения максимальной прибыли и оптимальную стратегию выхода на новые рынки сбыта.

\section{Список источников / References}

1. Семериков А. В. Решение транспортных задач. - Ухтинский государственный технический университет (УГТУ), 2013. - 58 с.

2. Рутковская Д., Пилиньский М., Рутковский Л. Нейронные сети, генетические алгоритмы и нечеткие системы $=$ Sieci neuronowe, algorytmy genetyczne i systemy rozmyte. - 2-е изд. - М: Горячая линия-Телеком, 2008. — 452 с. ISBN 5-93517- 103-1.

3. URL: https://pastebin.com/iDMddCYW

4. Писарук Н. Н., Исследование операций - Минск: БГУ, 2015. - 304 с.

5. Алексеева Е. В. Построение математических моделей целочисленного линейного программирования. Примеры и задачи: Учеб. Пособие / Новосиб. гос. ун-т. Новосибирск, 2012. $131 \mathrm{c}$.

6. Lee J., \& Wiegele A. (2017). Another pedagogy for mixed-integer Gomory. EURO Jour- nal on Computational Optimization, 5(4), 455-466. DOI: 10.1007/s13675-0170085-3

7. Xiaoping Jiang, Ruibin Bai, Jason Atkin, Graham Kendall. (2017) A scheme for determin- ing vehicle routes based on Arc-based service network design. INFOR: Information Sys- tems and Operational Research 55:1, pages 16-37.

8. Morrison D. R., Sewell E. C., \& Jacobson S. H. (2014). An application of the branch, bound, and remember algorithm to a new simple assembly line balancing dataset. European Journal of Operational Research, 236(2), 403-409.

9. Chu, W. S., de la Torre, F., Cohn, J. F., \& Messinger, D. S. (2017). A Branch-andBound Framework for Unsupervised Common Event Discovery. International Journal of Com- puter Vision, 1-20. DOI: 10.1007/s11263-017-0989-7

10. Siew Mooi Lim, Abu Bakar Md. Sultan, Md. Nasir Sulaiman, Aida Mustapha, and K. Y. Leong, "Crossover and Mutation Operators of Genetic Algorithms," International Journal of Machine Learning and Computing vol. 7, no. 1, pp. 9-12, 2017. 
11. P. Sumathi (2016) A new approach to solve linear programming problem with intercept values, Journal of Information and Optimization Sciences, 37:4, 495510, DOI: 10.1080/02522667.2014.996031

12. Daganzo, C. F., \& Smilowitz, K. R. (2004). Bounds and approximations for the transpor- tation problem of linear programming and other scalable network problems. Transportation Science, 38(3), 343-356. DOI: 10.1287/trsc.1030.0037

13. Hadi Heidari Gharehbolagh, Ashkan Hafezalkotob, Ahmad Makui, and Sedigh Raissi, "A cooperative game approach to uncertain decentralized logistic systems subject to network reliability considerations," Kybernetes, vol. 46, no. 8, pp. 1452-1468, 2017.

14. Maysara Sayed, Linda C. Hendry, Marta Zorzini Bell, (2017) "Institutional complexity and sustainable supply chain management practices", Supply Chain Management: An In- ternational Journal, Vol. 22 Issue: 6, pp.542-563, URL: https://doi.org/10.1108/ SCM-10- 2016-0365

\section{Сведения об авторах / About authors}

Рогулин Родион Сергеевич, магистрант направления «Прикладная математика и информатика», Дальневосточный федеральный университет. 690022 Россия, г. Владивосток, о-в Русский, кампус ДВФУ, корпус С. E-mail: rafassiaofusa@mail.ru

Rodion S. Rogulin, Master Student in Applied Mathematics and Computer Science, Far Eastern Federal University. Building C, FEFU campus, Russky Island, Vladivostok, Russia 690922.

E-mail: rafassiaofusa@mail.ru

Пугачёва Ева Сергеевна, студент направления «Медицинская биофизика», Дальневосточный федеральный университет. 690022 Россия, г. Владивосток, о-в Русский, кампус ДВФУ, корпус M. E-mail: pugacheva.es@students.dvfu.ru

Eva S. Pugacheva, Student of the Direction "Medical Biophysics", Far Eastern Federal University. Building M, FEFU campus, Russky Island, Vladivostok, Russia 690922.

E-mail: pugacheva.es@students.dvfu.ru

Матвеев Владислав Викторович, бакалавр направления «Биотехнология», Дальневосточный федеральный университет. 690022 Россия, г. Владивосток, о-в Русский, кампус ДВФУ, корпус M. E-mail: matveev.vvi@students.dvfu.ru

Vladislav V. Matveev, Bachelor of Biotechnology, Far Eastern Federal University. Building M, FEFU campus, Russky Island, Vladivostok, Russia 690922. E-mail: matveev.vvi@ students.dvfu.ru

Максименко Валерий Иванович, кандидат технических наук, доцент, доцент кафедры гидротехники, теории зданий и сооружений, Инженерная школа, Дальневосточный федеральный университет. 690022 Россия, г. Владивосток, о-в Русский, кампус ДВФУ, корпус С.

E-mail:maximenko.vi@dvfu.ru

Valeriy I. Maksimenko, Candidate of Technical Sciences, Associate Professor, Associate Professor of the Department of Hydraulic Engineering, Theory of Buildings and Structures, School of Engineering, Far Eastern Federal University. Building C, FEFU campus, Russky Island, Vladivostok, Russia 690922. E-mail: maximenko.vi@dvfu.ru

Жандармов Владимир Олегович, магистрант направления «Кибербезопасность», Дальневосточный Федеральный Университет. 690022 Россия, г. Владивосток, о-в Русский, кампус ДВФУ, корпус C. E-mail: zhandarmov.vo@students.dvfu.ru

Vladimir O. Zhandarmov, Master Student of Cybersecurity, Far Eastern Federal University. Building C, FEFU campus, Russky Island, Vladivostok, Russia 690922. E-mail: zhandarmov.vo@students.dvfu.ru 
Р.С. Рогулин и Ар. // Известия АВФУ. Экономика и управление. 3. 2019. 40-47

Рогулин Данила Сергеевич, студент направления «Прикладная информатика», Владивостокский государственный университет экономики и сервиса. 690014 Россия, г. Владивосток, ул. Гоголя, 41.

Danila S. Rogulin, Student of Applied Informatics, Vladivostok State University of Economics and Service. 41, Gogolya str., Vladivostok, Russia 690014.

( Р Рогулин Р.С., Пугачева Е.С., Матвеев В.В., Максименко В.И., Жандармов В.О., Рогулин Д.С.

C Rogulin R.S., Pugacheva E.S., Matveev V.V., Maksimenko V.I.., Zhandarmov V.O., Rogulin D.S

Адрес сайта в сети интернет: http://jem.dvfu.ru 